\title{
Mid-infrared parametric wavelength conversion seeded with fiber optical parametric sources
}

\author{
Ronan A. Battle ${ }^{1, *}$, Anita M. Chandran ${ }^{1}$, Timothy H. Runcorn ${ }^{1}$, Arnaud Mussot ${ }^{2}$, Alexandre Kudlinski ${ }^{2}$, Robert T. \\ Murray $^{1}$, and J. Roy Taylor ${ }^{1}$ \\ ${ }^{1}$ Femtosecond Optics Group, Department of Physics, Imperial College London, Prince Consort Road, London SW7 2BW, UK \\ ${ }^{2}$ Université de Lille, CNRS, UMR 8523-PhLAM_Physique des Lasers Atomes et Molécules, F-59000 Lille, France
}

\begin{abstract}
A new method of seeding $\chi^{2}$ optical parametric converters with $\chi^{3}$ fiber optical parametric sources is introduced. We demonstrate a tunable mid-infrared source operating at around $3 \mu \mathrm{m}$ with the technique and discuss the potential of this architecture.
\end{abstract}

\section{Introduction}

The mid-infrared (MIR) spectral region contains strong characteristic molecular absorption features of great interest for a range of applications. Directly emitting MIR sources in this region are limited, but near-infrared (NIR) solid-state lasers can be used to generate MIR radiation through various $\chi^{(2)}$ parametric three-wave mixing (3WM) conversion techniques in nonlinear crystals [1]. Optical parametric amplification (OPA), and difference frequency generation (DFG) are examples of simple 3WM techniques which provide significant flexibility in output repetition rates, pulse durations, and wavelengths. However, a seed signal at the correct wavelength, synchronised with the pump laser, is also required, which can necessitate the use of expensive secondary lasers or complex nonlinear conversion stages. The pump and signal beams must also be precisely spatially overlapped in the crystal. These two requirements result in increased system complexity, and can lead to such sources being difficult to use by non-experts, restricting uptake in clinical and/or field applications.

In this contribution, we introduce and demonstrate a novel method of seeding $\chi^{2}$ optical parametric converters with $\chi^{3}$ fiber optical parametric sources, illustrated in Fig. 1(a). By pumping optical fibers in the low normal dispersion region, it is possible to generate widely spaced, high power spectral density Stokes/anti-Stokes pulses through four-wave mixing (4WM) [2]. These Stokes pulses are inherently temporally and spatially overlapped with the pump pulse at the exit of the fiber, provided the walk-off length is long compared to the length of fiber employed. The output of the fiber can therefore be focused directly into a nonlinear crystal to convert the pump and Stokes/anti-Stokes pulses into the MIR through 3WM. Consequently, the need for additional signal beam

*e-mail: ronan.battle15@imperial.ac.uk delay and combination optics - commonly required in OPA and DFG systems - is avoided.

The use of 4WM in PCF to generate the signal pulses required for $3 \mathrm{WM}$ has previously been explored by Yao and Knox [3]. However, they used pump pulses with a duration of $300 \mathrm{fs}$ and a double zero dispersion wavelength (ZDW) PCF, neither of which are suitable for the architecture described here, owing to the large walk-off resulting from such short pump pulses, and the limited coupling efficiencies into the PCF. The single ZDW PCFs and longer pump pulse durations ( $35 \mathrm{ps}$ ) used in this work are key to enabling the direct focusing of the PCF output into the crystal. As a result, the architecture we demonstrate here could be fully fiber integrated [4], resulting in a compact, robust, and integrated pump system for the $3 \mathrm{WM}$ stage. This could ultimately lead to more user-friendly and fielddeployable MIR sources, useful for a range of applications from surgery to remote sensing. We used the architecture to generate picosecond MIR pulses with pulse energies up to $\sim 14 \mathrm{~nJ}$ and average powers up to $115 \mathrm{~mW}$. The pulses were wavelength tunable around $3 \mu \mathrm{m}$, coinciding with the water absorption peak at $2.94 \mu \mathrm{m}$, making them well suited for biomedical laser ablation applications [5].

\section{Experiment and Discussion}

To demonstrate the technique, we first selected an optical fiber and nonlinear crystal with a common Stokes and signal wavelength, as highlighted in the $4 \mathrm{WM}$ and $3 \mathrm{WM}$ phase matching curves in Fig. 1(b) and Fig. 1(c). Pumping these nonlinear media at $1.064 \mu \mathrm{m}$ results in a common Stokes and signal wavelength around $1.65 \mu \mathrm{m}$. Thus, the Stokes pulses generated in the $4 \mathrm{WM}$ stage can be used as the seed signal in the 3WM stage as illustrated in Fig. 1(a).

We employed an in-house developed ytterbium fiber master oscillator power amplifier (Yb:fiber MOPA) system shown in Fig. 2 as the pump source. The MOPA system consisted of a $50 \mathrm{MHz}$ mode-locked fiber oscillator 


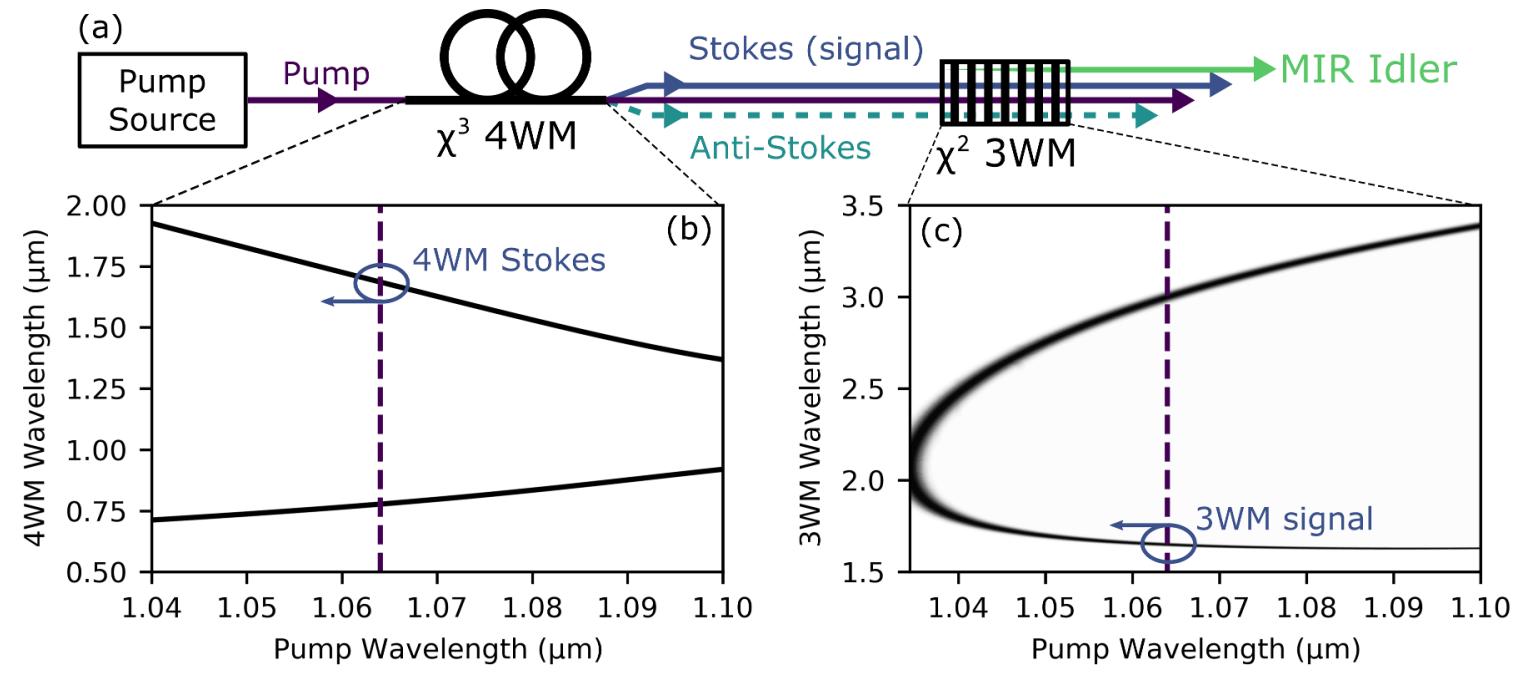

Figure 1. (a) Schematic of MIR generation via cascaded $\chi^{(3)} 4 \mathrm{WM}$ in fibre and $\chi^{(2)} 3 \mathrm{WM}$ in a bulk non-linear crystal. (b) Phase matching diagrams for the 4WM in PCF and (c) $3 \mathrm{WM}$ in PPLN (at $60^{\circ} \mathrm{C}$ ). Circles highlight the common wavelength which is phase matched in both the $4 \mathrm{WM}$ and $3 \mathrm{WM}$ interactions.

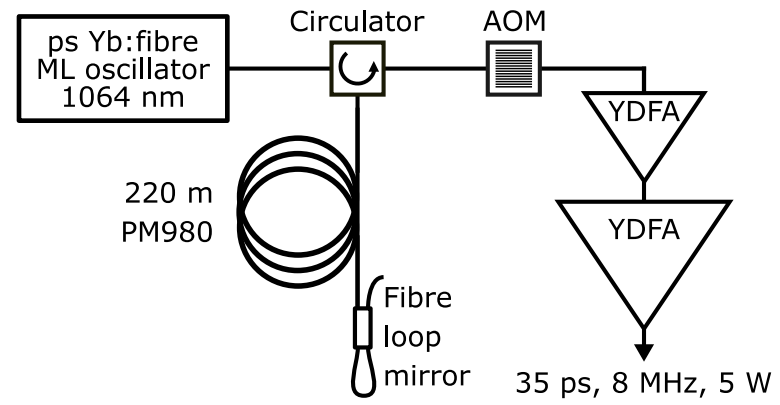

Figure 2. In-house developed ytterbium fiber master oscillator power amplifier system. ML, mode-locked; AOM, acousto-optic modulator; YDFA, ytterbium-doped fibre amplifier.

that produced 5 ps pulses, stretched to 35 ps in a double pass of $220 \mathrm{~m}$ of polarization maintaining fibre (PM980, Newport), picked down to $\sim 8 \mathrm{MHz}$ by an acousto-optic modulator (AOM) and amplified to $5 \mathrm{~W}$ in two ytterbiumdoped fibre amplifiers (YDFA). The output spectrum of the MOPA, which has a 10-dB spectral width of $7.1 \mathrm{~nm}$, is shown in Fig. 3(a). A long pass $1100 \mathrm{~nm}$ filter was used before the PCF to filter out light at $1120 \mathrm{~nm}$ resulting from stimulated Raman scattering in the final amplifier stage.

The custom polarization-maintaining photonic crystal fiber (PCF) used had zero dispersion wavelengths of $1.101 \mu \mathrm{m}$ and $1.103 \mu \mathrm{m}$ on the fast and slow axes respectively [6]. Pumping a $0.35 \mathrm{~m}$ length of PCF on the slow axis with $3.1 \mathrm{~W}$ of average power (coupled), we generated $1.65 \mu \mathrm{m}$ Stokes and $0.785 \mu \mathrm{m}$ anti-Stokes sidebands shown in Fig. 3(b). The average powers generated were $150 \mathrm{~mW}$ (Stokes) and $260 \mathrm{~mW}$ (anti-Stokes).

The output of the PCF was then focused directly into a periodically poled lithium niobate crystal doped with $5 \mathrm{~mol} \% \mathrm{MgO}$ (PPLN, HC Photonics) to generate signal and idler light through $3 \mathrm{WM}$. The $3 \times 3 \times 10 \mathrm{~mm}^{3}$ crystal used had a poling period of $31.3 \mu \mathrm{m}$. The PPLN was held in a crystal oven to enable temperature tuning in the range $20-200^{\circ} \mathrm{C}$. The maximum pump peak intensity in the crystal was estimated to be $1.7 \mathrm{GW} / \mathrm{cm}^{2}$, calculated from a measured spot size of $35 \mu \mathrm{m}$ at focus. The spatial overlap of the pump and Stokes pulses at the focus was confirmed using a scanning slit beam profiler. Due to the long walkoff length $(2.0 \mathrm{~m})$ between the pump and Stokes pulses relative to the short PCF length employed $(0.35 \mathrm{~m})$, the pulses were assumed to be well overlapped temporally for the $3 \mathrm{WM}$ stage.

As the Stokes seed generated in the 4WM stage was spectrally broader than the 3WM spectral acceptance bandwidth, tuning of the crystal temperature in the range $40-100^{\circ} \mathrm{C}$ resulted in tuning of the idler spectra as shown in Fig. 3(c). The associated variation in the generated idler and output signal powers are shown in Fig. 3(d). Moving away from the temperature at which the seed signal was phase matched resulted in no idler generation; no (spontaneous) optical parametric generation was observed. This confirmed that the pulses we generated in the 4WM stage were seeding the $3 \mathrm{WM}$. A maximum $115 \mathrm{~mW}$ of generated $3 \mathrm{WM}$ idler alongside $189 \mathrm{~mW}$ of amplified 3WM signal was generated at a crystal temperature of $60^{\circ} \mathrm{C}$. This corresponds to a pump conversion efficiency of $11 \%$ (amplified signal plus idler) in the $3 \mathrm{WM}$ stage.

The $3 \mathrm{WM}$ conversion efficiency was primarily limited by the broad bandwidth of the pump and signal relative to the spectral acceptance bandwidths of the crystal. Employing a narrower bandwidth pump/signal would allow an increase in conversion in the $3 \mathrm{WM}$ stage. This could be readily achieved by altering the pump laser parameters (bandwidth and pulse duration) and adjusting the length of the fiber in the 4WM stage.

In a system such as this, however, which employs cascaded nonlinear effects, various competing factors must be considered. In the 4WM stage, sufficient Stokes power must be generated to seed the subsequent 3WM. Ideally, 
(a)

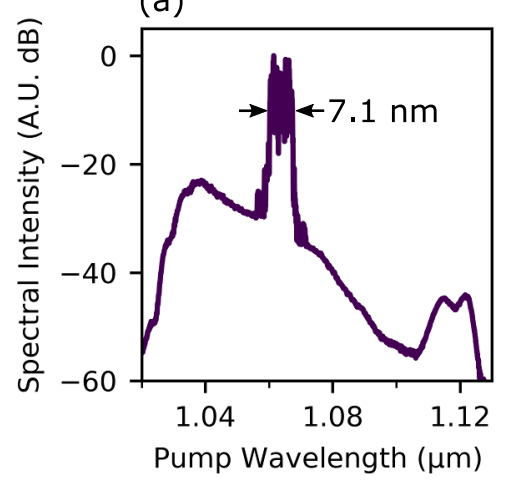

(c)

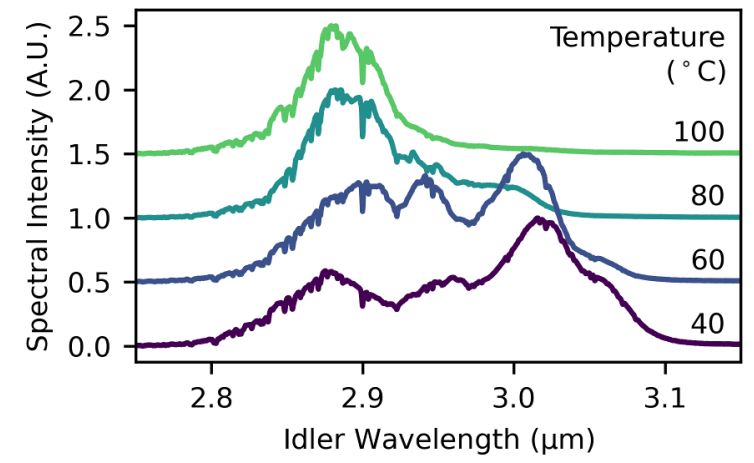

(b)

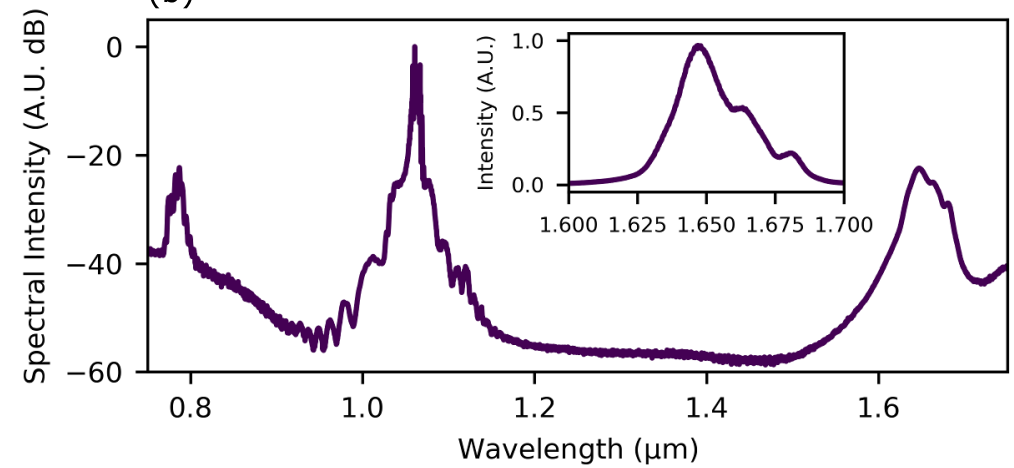

(d)

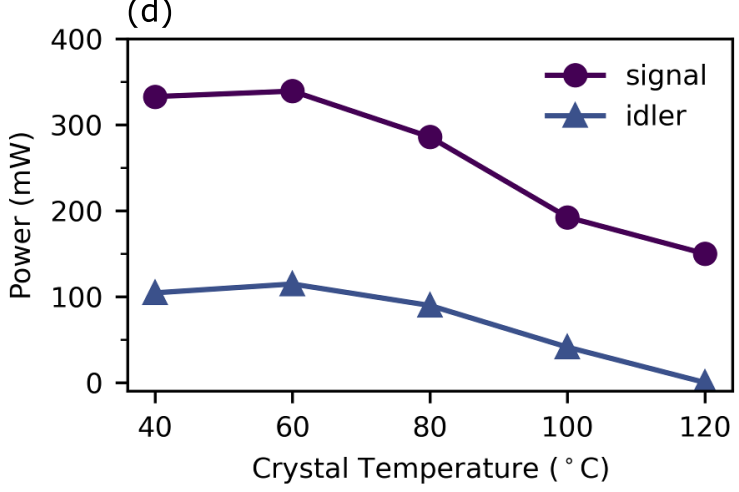

Figure 3. (a) MOPA pump laser spectrum centered at $1.064 \mu \mathrm{m}$, with bandwidth of $7.1 \mathrm{~nm}$. (b) Spectrum at output of PCF showing $1.064 \mu \mathrm{m}$ pump and 4WM sidebands. Inset: Stokes sideband on linear scale. (c) Idler spectral tuning with crystal temperature on linear scale (d) Generated signal and idler powers as a function of temperature.

the Stokes power generated would saturate the $3 \mathrm{WM}$ to achieve the greatest output power and stability in the generated MIR idler power [7]. Conversely, increased pump conversion to the Stokes pulses in the 4WM stage results in less residual pump power remaining to pump the $3 \mathrm{WM}$ stage, limiting the $3 \mathrm{WM}$ conversion. As discussed, the pump bandwidth must also not exceed the $3 \mathrm{WM}$ pump spectral acceptance bandwidth. Some self-phase modulation induced pump broadening is unavoidable in the $4 \mathrm{WM}$ stage, because the pump must propagate through normally dispersive fiber to generate the required 4WM sidebands. Ongoing work aims to balance these cascading nonlinearities to ascertain the performance limits of this architecture.

\section{Conclusion}

We have introduced and demonstrated a new method of seeding $\chi^{2}$ parametric wavelength converters $(3 \mathrm{WM})$ directly with $\chi^{3}$ fiber optical parametric sources (4WM). By exploiting the inherent spatial and temporal overlap of pulses generated by $4 \mathrm{WM}$ in PCF, we seed $3 \mathrm{WM}$ in PPLN to generate $115 \mathrm{~mW}$ at $\sim 3 \mu \mathrm{m}$. Further investigations and simulations are ongoing to determine the limits of the conversion efficiency achievable using this architecture.

\section{References}

[1] V. Petrov, in Progress in Quantum Electronics (Elsevier Ltd, 2015), Vol. 42, pp. 1-106

[2] L. Lavoute, P.J. Mosley, S.A. Bateman, W.J. Wadsworth, Opt. Express 19, 25337 (2011)

[3] Y. Yao, W.H. Knox, Opt. Express 20, 25275 (2012)

[4] M. Baumgartl, M. Chemnitz, C. Jauregui, T. Meyer, B. Dietzek, J. Popp, J. Limpert, A. Tünnermann, Opt. Express 20, 4484 (2012)

[5] S. Amini-Nik, D. Kraemer, M.L. Cowan, K. Gunaratne, P. Nadesan, B.A. Alman, R.J.D. Miller, PLoS ONE 5, e13053 (2010)

[6] R.T. Murray, E.J.R. Kelleher, S.V. Popov, A. Mussot, A. Kudlinski, J.R. Taylor, Opt. Express 21, 15826 (2013)

[7] F. Mörz, T. Steinle, A. Steinmann, H. Giessen, Opt. Express 23, 23960 (2015) 